\title{
A NOTE TO THE PAPER ON INTEGRAL BASES BY H. B. MANN
}

VIRGINIA HANLY AND H. B. MANN

The definitions and notation are those of (2). We shall prove the following theorem.

THEOREM. If $\Im$ contains an ideal which is not principal then there exists a quadratic extension $\mathfrak{F}^{\prime}$ over $\mathfrak{F}$ which has no integral basis over $\mathfrak{F}$.

The theorem was proved in (2) for the case that $\mathfrak{F}$ has characteristic different from 2 . Here we shall give the proof in the case that $\mathfrak{F}$ has characteristic 2 .

Let $\mathfrak{F}$ have characteristic 2 and let $\mathfrak{F}^{\prime}=\mathfrak{F}(\theta)$ where $\theta^{2}+b \theta+c=0$; $b, c \in \Im, b \neq 0$. Every $\alpha \in \mathfrak{F}^{\prime}$ may be written as $y_{0}+y_{1} \theta ; y_{0}, y_{1} \in \mathfrak{F}$. If $\alpha$ is integral then $T(\alpha)=b y_{1}$ and $T(\alpha \theta)+y_{1} b^{2}=b y_{0}$ are integers. Hence we may write

$$
\alpha=\frac{x_{0}+x_{1} \theta}{b} ; \quad x_{0}, x_{1} \in \Im
$$

The integers $x_{1}$ appearing in these representations form an ideal $a$ of $J$.

Lemma 1. We have $\mathfrak{a}=\mathfrak{d}$, where $\mathfrak{b}$ is the different of $\mathfrak{F}^{\prime}$ over $\mathfrak{F}$.

Proof. The ideal $a$ is the g.c.d. of all traces of elements in $J^{\prime}$. Hence $\mathfrak{b} \equiv 0(\bmod \mathfrak{a})$. On the other hand $\mathfrak{a}$ is also the g.c.d. of all differents of elements of $\Im^{\prime}$ since $\mathfrak{F}$ has characteristic 2 . Hence $a \equiv 0(\bmod d)$ and so $\mathfrak{a}=\mathfrak{b}$. (The proof of Lemma 11.5.1 of (1) carries over without change to any Dedekind ring.)

Lemma 2. Let $\mathfrak{F}$ have characteristic 2 and let $\mathrm{q}$ be any squarefree ideal of $\mathfrak{\Im}$. There exists a quadratic extension $\mathfrak{F}^{\prime}$ over $\mathfrak{F}$ whose different over $\mathfrak{F}$ is $\mathfrak{q}$.

Proof. We determine $q^{\prime}$ so that $\left(q^{\prime}, q\right)=1$ and $q^{\prime} q=(b), b \in \mathcal{F}$ and $q^{\prime \prime}$ so that $\left(b, \mathfrak{q}^{\prime \prime}\right)=1$ and $\mathfrak{q}^{\prime \prime} \mathfrak{q}=(c), c \in \Im$ and $c \equiv b+1\left(\bmod \mathfrak{q}^{\prime 2}\right)$. The polynomial $x^{2}+b x+c$ is irreducible by Eisenstein's criterion. If $\theta$ is one of its roots then $\alpha=(y+\theta x) / b, x, y \in \mathcal{Y}$ is integral if and only if $N(\alpha)$ is integral, hence if and only if

$$
x^{2} c+b x y+y^{2} \equiv 0\left(\bmod b^{2}\right) .
$$

By Lemma $1 \delta$ is the g.c.d. of all $x$ for which (1) has a solution $y \in \Im$.

Received by the editors May 28, 1957. 
We then have $y \equiv 0(\bmod \mathfrak{q})$, hence $x \equiv 0(\bmod \mathfrak{q})$. On the other hand for $x=y \equiv 0(\bmod \mathfrak{q})$ we have by our construction of $b$ and $c$

$$
\begin{aligned}
& x^{2}(c+b+1) \equiv 0\left(\bmod \mathfrak{q}^{2}\right), \\
& x^{2}(c+b+1) \equiv 0\left(\bmod \mathfrak{q}^{\prime 2}\right),
\end{aligned}
$$

whence $x^{2}(c+b+1) \equiv 0\left(b^{2}\right)$ and therefore $\mathfrak{b}=q$.

Our theorem now follows easily from Lemma 2 and Theorem 5 of (2).

\section{REFERENCES}

1. H. B. Mann, Introduction to algebraic number theory, The Ohio State University Press, Columbus, 1955.

2. - On integral bases, Proc. Amer. Math. Soc. vol. 9 (1958) pp. 167-172.

The Ohio State University 